\section{Chewing gum added to fresh breath kit}

The Breath Co. have launched their Fresh Breath Chewing Gum in Boots stores and online ( $£ 4$ per pack).

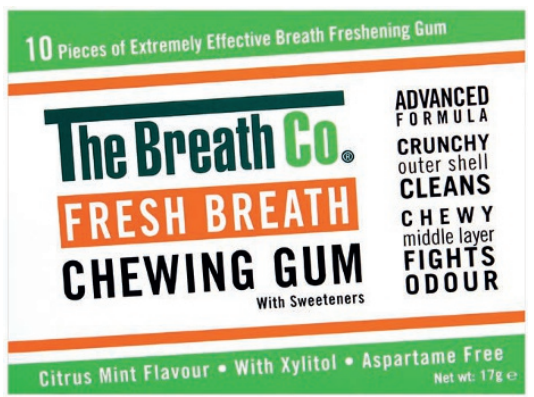

Until now, the only way to stop bad breath was to mask odour. The Breath Co. Gum is different. It does not mask odours with strong scents or flavourings. It fights them by attacking and neutralising volatile sulphur compounds (VSCs) that cause unpleasant breath odours. This gum is even effective on odours caused by onions, garlic, coffee and smoking. It will leave your whole mouth refreshed and your confidence restored.

This is an extension to The Breath Co.'s current product line of bad breath beating products - the oral-wash, lozenges and toothpaste. Boots offer all products individually and The Breath Co. Starter Kit which is $£ 20$ and includes the chewing gum, oral rinse and toothpaste.

\section{Clustered glass fibre-reinforced composite root post}

Rebilda Post GT is a radiopaque, translucent, glass fibre-reinforced composite root post which exhibits dentine-like elasticity and ensures a considerably stronger bond to the core build-up than is achieved by conventional root posts. Unlike other post systems, Rebilda Post GT consists of a cluster of fine individual posts which are initially held together by a sleeve and thus can be inserted into the root canal in one working step.

Rebilda Post GT allows a customised and at the same time substance-preserving restoration, as the fine individual posts can be distributed throughout the entire root canal following removal of the sleeve and then adapt optimally to the respective root canal morphology. Root canal enlargement with a drill for a correspondingly sized post is thus not necessary, meaning that no additional tooth substance is lost, which would inevitably result in weakening of the root structure. Its unique composition makes Rebilda Post GT particularly suitable for use in non-round root canals and in root canals prepared using a larger cone in the greater taper technique, from which the 'GT' in the product name is derived.

Following pre-treatment of the root canal, the cluster of posts is silanised, coated with luting composite and then inserted into the root canal filled with luting composite. Prior to polymerisation of the luting composite, the sleeve is removed, so that the individual posts can be fanned out throughout the canal using a suitable instrument. This spreading out of the posts leads to both homogeneous reinforcement of the luting composite throughout the length of the root canal and an increase of the contact surface with the build-up composite in the coronal region, which results in a high degree of stability for the post/build-up system overall.

Rebilda Post GT is available in four colour-coded sizes: No. 4 (blue, idealised diameter of $0.8 \mathrm{~mm}$ ) containing 4 , No. 6 (red, idealised diameter of 1.0 $\mathrm{mm}$ ) containing 6, No. 9 (green, idealised diameter of $1.2 \mathrm{~mm}$ ) containing 9 and No. 12 (black, idealised diameter of $1.4 \mathrm{~mm}$ ) containing 12 individual posts. www.voco.com

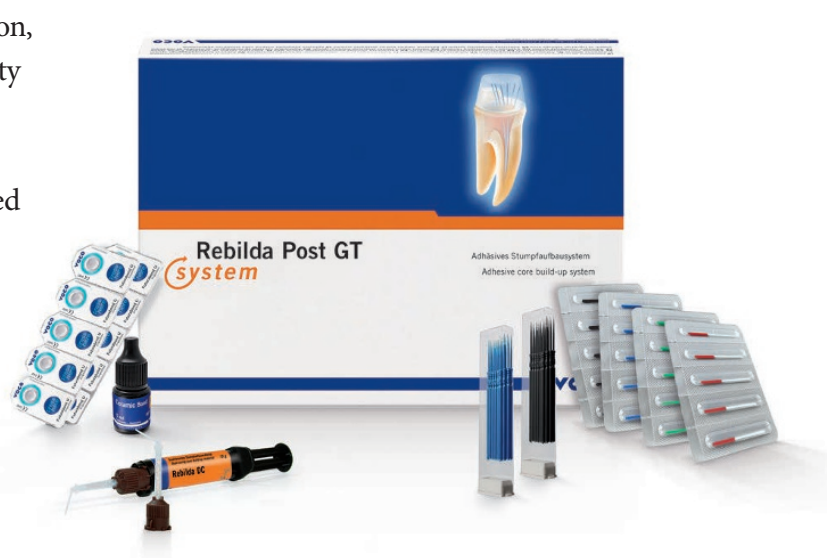

\title{
Improve recall effectiveness
}

Patient recalls are often forgotten or sent only once in the hope the patient receives the message first time. Missed appointment patients are not contacted for re-booking and as a result end up in an 'idle' state with no activity in the practice. All of these issues lead to large lists of patients being registered but not being treated.

Understanding and reacting to the needs of patients is fundamental to proactive marketing. As a recognition of these issues, iSmile has a built-in Campaign Manager which can automatically generate up to three rounds of appointment reminders and up to eight rounds of recall reminders. Each message is set to be sent via any channel (SMS, email, letter or patient preference) and the message for each round of reminders can be customised. With all the activity happening automatically in the background it eases pressure on practice managers and receptionists and ensures the practice knows the state of each registered patient.

Furthermore, the iSmile system extends directly to the palm of your patients. The digital age has created a connected world

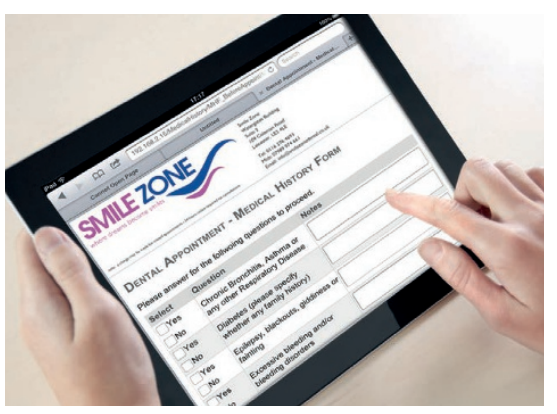

with constant access to key information anywhere, anytime on any device. iSmile has an integrated Patient Portal, which can be accessed from any device and is branded to the practice's specifications. The secure Patient Portal enables the patient to complete their medical history ahead of their appointment, minimising surgery wait times and allowing the dentist to see more patients during the day.

The iSmile Patient Portal also captures patient feedback on their experience and includes a patient interest form. The feedback can then be analysed at the practice and interest specific campaigns can be setup.

For more information about iSmile call 08454681287 or visit www.ismiledental.co.uk. 